\title{
Biomechanical evaluation of straight antegrade nailing in proximal humeral fractures: the rationale of the "proximal anchoring point"
}

\author{
Simon A. Euler ${ }^{1,2,3} \cdot$ Maximilian Petri ${ }^{2,3,4}$ - Melanie B. Venderley ${ }^{3} \cdot$ Grant J. Dornan $^{3}$. \\ Werner Schmoelz $^{1}$ • Travis Lee Turnbull ${ }^{3}$. Michael Plecko ${ }^{5}$ - Franz S. Kralinger ${ }^{6}$. \\ Peter J. Millett ${ }^{2,3}$
}

Received: 11 November 2016/Accepted: 24 April 2017 /Published online: 11 May 2017

(C) The Author(s) 2017. This article is an open access publication

\begin{abstract}
Purpose Varus failure is one of the most common failure modes following surgical treatment of proximal humeral fractures. Straight antegrade nails (SAN) theoretically provide increased stability by anchoring to the densest zone of the proximal humerus (subchondral zone) with the end of the nail. The aim of this study was to biomechanically investigate the characteristics of this "proximal anchoring point" (PAP). We hypothesized that the PAP would improve stability compared to the same construct without the PAP.

Methods Straight antegrade humeral nailing was performed in 20 matched pairs of human cadaveric humeri for a simulated unstable two-part fracture.

Results Biomechanical testing, with stepwise increasing cyclic axial loading (50-N increments each 100 cycles) at an angle of $20^{\circ}$ abduction revealed significantly higher median loads to
\end{abstract}

Investigation performed at the Department of BioMedical Engineering at the Steadman Philippon Research Institute, Vail, Colorado

Simon A. Euler

simon.euler@tirol-kliniken.at

1 Department of Trauma Surgery and Sports Traumatology, Medical University Innsbruck, Anichstr. 35, 6020 Innsbruck, Austria

2 The Steadman Clinic, 181 West Meadow Drive, Suite 400, Vail, CO 81657, USA

3 Steadman Philippon Research Institute, 181 West Meadow Drive, Suite 1000, Vail, CO 81657, USA

4 Department of Trauma Surgery, Hannover Medical School, Carl-Neuberg-Str. 1, 30625 Hannover, Germany

5 Unfallkrankenhaus Graz, Göstinger Str. 24, 8020 Graz, Austria

6 Department of Trauma Surgery, Wilhelminenspital, Montlearstr. 37 , 1160 Vienna, Austria failure for SAN constructs with the PAP (median, $450 \mathrm{~N}$; range, 200-1.000 N) compared to those without the PAP (median, $325 \mathrm{~N}$; range, $100-500 \mathrm{~N} ; p=0.009$ ). SAN constructs with press-fit proximal extensions (endcaps) showed similar median loads to failure (median, $400 \mathrm{~N}$; range, 200-650 N), when compared to the undersized, commercially available SAN endcaps (median, $450 \mathrm{~N}$; range, $200-600 \mathrm{~N} ; p=0.240$ ).

Conclusions The PAP provided significantly increased stability in SAN constructs compared to the same setup without this additional proximal anchoring point. Varus-displacing forces to the humeral head were superiorly reduced in this setting. This study provides biomechanical evidence for the "proximal anchoring point's" rationale. Straight antegrade humeral nailing may be beneficial for patients undergoing surgical treatment for unstable proximal humeral fractures to decrease secondary varus displacement and thus potentially reduce revision rates.

Keywords Humeral fracture $\cdot$ Straight antegrade nails . Proximal anchoring point $\cdot$ Stability

\section{Introduction}

Currently, no consensus has been reached on the surgical treatment of displaced or unstable proximal humeral fractures. Complication and revision rates remain high. In up to $25 \%$ of cases, post-operative loss of reduction and varus failure have been frequently described as two of the most common failure modes associated with surgical stabilization [1-3]. As an alternative surgical option to lateral locking-plate fixation, straight antegrade humeral nailing (SAN) has been introduced, providing promising results clinically $[1,4]$. Biomechanically, the lever arm of an intramedullary device is lower compared with a lateral locking plate, potentially counteracting varus displacing forces 
superiorly. In contrast to the bent proximal humeral nail, the straight designed nail appears to provide several additional advantages (please also see Table 1):

- The medialization of the entry point preserves the supraspinatus tendon footprint (Fig. 1) [5].

- The medialization of the proximal part of the nail within the humeral head preserves a larger amount of bony substance lateral to the nails rim ("lateral bony bridge"), additionally increasing the constructs capability to resist varus displacing forces (Fig. 1).

- In cases of proximal humeral fractures which include a fractured greater tuberosity, inadvertent entry to the fracture zone may be prevented (Fig. 1b).

- The straight nails proximal tip is designed to anchor the densest subchondral zone at the top of the humeral head, potentially counteracting varus displacing forces thus providing enhanced stability [6] (Fig. 1a).

Although this concept of the "proximal anchoring point" (PAP) has been described in the literature, its potential biomechanical advantage has never been biomechanically proven [5].

The aim of this study was to investigate the biomechanical characteristics of the PAP and to validate a component of the SAN's technical rationale. We hypothesized that the additional anchoring point in SAN for proximal humeral fractures with comminuted medial calcar would significantly improve stability compared to the same construct without the PAP.

\section{Materials and methods}

This study was performed in accordance with the ethical standards as laid down in the 1964 Declaration of Helsinki and its later amendments or comparable ethical standards.

\section{Specimen preparation}

Twenty matched pairs ( $n=40)$ of fresh-frozen human cadaveric shoulders (ten male, ten female; mean age, 82.7 years [range, 73-95]) were utilized for this study. Paired specimens were randomly distributed between two homogenous groups: group A, no PAP (five male, five female; mean age, 82.6 years [range, 73-92]; mean BMI 23.1 [range, 15.4-33.4]; mean BMD $67 \mathrm{mg} / \mathrm{cm}^{3}$ [range, 52-83]) and the group B, custom PAP (five male, four female; mean age, 81.9 years [range, 7595]; mean BMI 25.2 [range, 18.8-34.7]; mean BMD $73 \mathrm{mg}$ / $\mathrm{cm}^{3}$ [range, 55-88]) from highest to lowest age. The first pair was randomly assigned by flipping a coin. There were no significant differences between the groups regarding age, gender, BMI or BMD. Within no-PAP group A, subgroup A1 was assigned a common endcap (undersized, compared to the nail's proximal tip diameter) attached to the tip of the nail, while subgroup A2 did not have an endcap. Within customPAP group B, subgroup B1 was assigned a common endcap (identical construct as subgroup A1), while subgroup B2 was given a custom endcap (equally sized, compared to the nail's proximal tip diameter) (Fig. 2). One pair of humeri (group B) had to be excluded from final analysis due to incorrect potting of one humeral head, leading to instability during biomechanical testing.

To estimate the bone mineral density (BMD), computed tomography (CT) scans of all specimens were conducted. According to the method described by Krappinger et al. [7], the cancellous BMD of the proximal humeral heads, corrected for yellow fat marrow, were calculated.

Prior to preparation, specimens were thawed at room temperature for 24 hours and dissected free of all soft tissue. By visual and $\mathrm{CT}$ scan inspection, no prior surgeries, preexisting injuries or pathologies, other than osteoporosis were identified. According to the age, some paired specimens showed mild to severe grades of osteoarthritis.

Table 1 Biomechanical properties and comparison of the most common concepts for the reconstructive surgical treatment of proximal humeral fractures. Please also see Fig. 1 for further explanation and illustration

\begin{tabular}{|c|c|c|c|c|}
\hline Concept & Entry point & $\begin{array}{l}\text { Anchoring points } \\
\text { within the humeral head }\end{array}$ & Strengths & Limitations \\
\hline $\begin{array}{l}\text { Straight } \\
\text { humeral } \\
\text { nail }\end{array}$ & $\begin{array}{l}\text { Apex (center) of the } \\
\text { humerus }\end{array}$ & $\begin{array}{l}\text { Tip of the nail ("proximal } \\
\text { anchoring point") } \\
\text { - Locking screws }\end{array}$ & $\begin{array}{l}\text { Potential superior resistance } \\
\text { to varus forces } \\
\text { Strong and wide lateral bony } \\
\text { bridge } \\
\text { Minimal invasive }\end{array}$ & $\begin{array}{l}\text { Ideal entry point difficult to define in case } \\
\text { of severe dislocation }\end{array}$ \\
\hline $\begin{array}{l}\text { Bent humeral } \\
\text { nail }\end{array}$ & $\begin{array}{l}\text { Transition zone (greater } \\
\text { tuberosity) }\end{array}$ & Locking screws & Minimal invasive & $\begin{array}{l}\text { Entry point potentially in the fracture line } \\
\text { (three-part fracture including the greater } \\
\text { tuberosity) } \\
\text { Potential violation of the Supraspinatus } \\
\text { footprint } \\
\text { (region of tendon nutrition) }\end{array}$ \\
\hline Locking plate & n.a. (lateral) & Screws & Cement augmentation option & Relatively large surgical approach \\
\hline
\end{tabular}



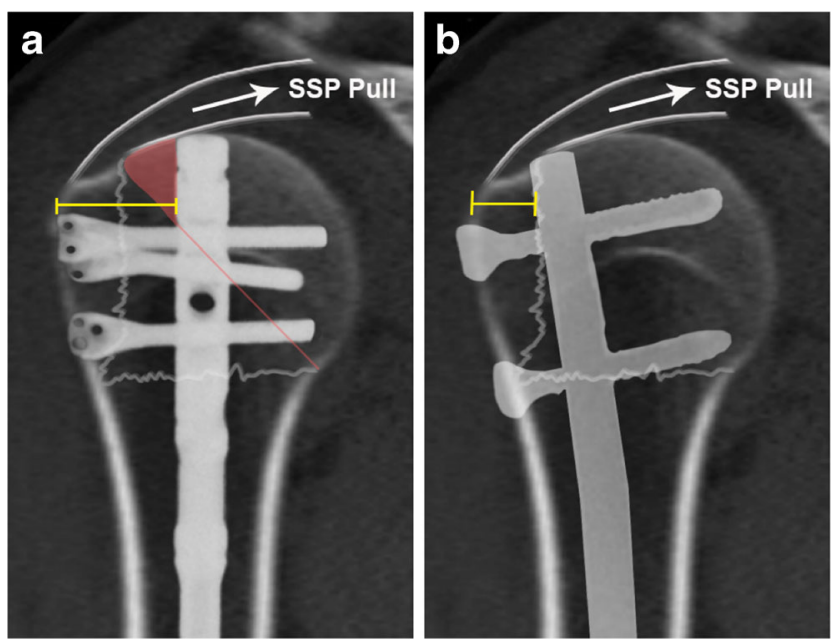

Fig. 1 Schematic illustration of the most important biomechanical properties of straight and bent antegrade humeral nails. a Anterior/ posterior view on a proximal humerus with a straight antegrade humeral nail implanted. b Bent antegrade humeral nail. Yellow dimension line lateral bony bridge, red triangle bony substance counteracting varus displacing forces, SSP supraspinatus tendon

The humeri were cut distally to a length of $175 \mathrm{~mm}$ and potted to a depth $135 \mathrm{~mm}$ distal to the humeral head's most proximal extension in polymethyl methacrylate (PMMA; Fricke Dental International, Streamwood, IL, USA) using a custom cylindrical mold, with the long axis of the humerus concentric with the cylindrical axis of the mold. Prior to potting, screws were inserted through the shaft into the most distal $5 \mathrm{~mm}$ of the humeri to ensure rigid fixation in the PMMA.

According to the manufacturer's technique guide (DePuy Synthes, West Chester, PA, USA), the dedicated entry points of the nails on the humeral heads were identified and marked in line with the shaft axis.

\section{Surgical technique}

The SAN's entry point was determined by projection of the physiological shaft axis at the top of the head fragment. The head was then opened using an $11.5-\mathrm{mm}$ drill, and the nail (MultiLoc $9.5 \times 160 \mathrm{~mm}$, proximal diameter $11 \mathrm{~mm}$; DePuy Synthes, West Chester, PA, USA) was implanted. MultiLoc proximal locking screws (4.5-mm MultiLoc Titanium; DePuy Synthes, West Chester, PA, USA) of the appropriate length were inserted into the humeral head, according to the manufacturer's technique guide. The locking screws were chosen to be as long as possible but to not penetrate the humeral cortex contralaterally, and were placed in the same standardized orientation for every specimen.

Two bicortical antirotational locking screws (4.0-mm Titanium Locking; DePuy Synthes, West Chester, PA, USA) were inserted distally. All screws were placed using the manufacturer's aiming frame. To ensure comparable positioning of the locking screws between pairs, the nail's proximal tip was distalized $10 \mathrm{~mm}$ below the most superior extent of the humeral head. Although this seems to appear rather distal in some cases, it does reflect clinical reality - in tall individuals, the nail's tip will end up to $15 \mathrm{~mm}$ distal to the humeral head's most proximal cortical extension in order to place the very proximal and distal locking screw options correctly. An endcap (MultiLoc Endcap, diameter $9.5 \mathrm{~mm}$ [only available diameter], length $10 \mathrm{~mm}$; DePuy Synthes, West Chester, PA, USA) was attached to one humerus from each pair (subgroups A1 and B1). The contralateral humerus nail's proximal tip was left uncapped in the no-PAP group (subgroup A2), while a 10$\mathrm{mm}$-long custom endcap with a diameter of $11 \mathrm{~mm}$ (in accordance with the proximal nail's diameter) was attached in the custom-PAP group (subgroup B2).

Finally, a 10-mm circumferential osteotomy was created in all specimens to imitate a two-part proximal humeral fracture with a comminuted medial calcar. The proximal cut for the osteotomy was initiated $2 \mathrm{~mm}$ below the most distal level of the articular cartilage. All specimens were then potted in a custom cylindrical mold to a depth of approximately $40 \mathrm{~mm}$, so as to completely cover the articular cartilage. According to previously described models, the long axis of the humerus was oriented in neutral position and $20^{\circ}$ of abduction relative
Fig. 2 Testing groups.

Animation of nail configurations in a cadaveric osteoporotic humerus (anterior view). $A 2$ nail without endcap; $A 1$ and $B 1$ nail with the common undersized endcap; $B 2$ nail with the custom endcap, equal to the nails proximal diameter. Black circle indicating the "proximal anchoring point"

\section{Testing Groups}

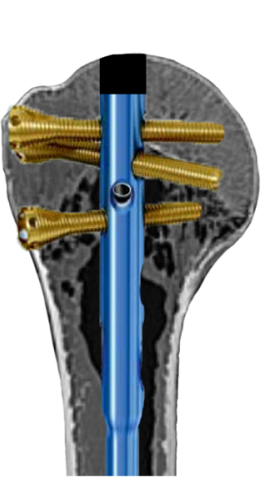

A2

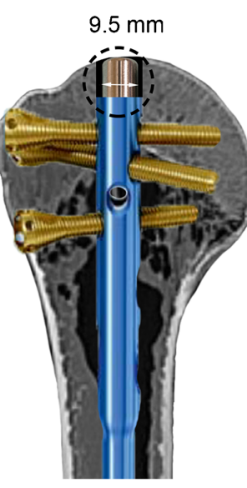

A1

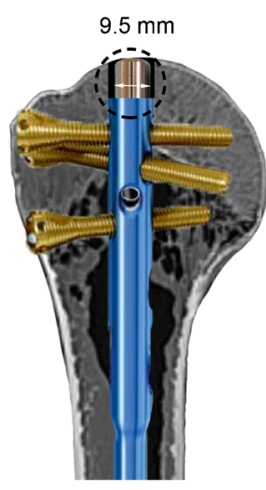

B1

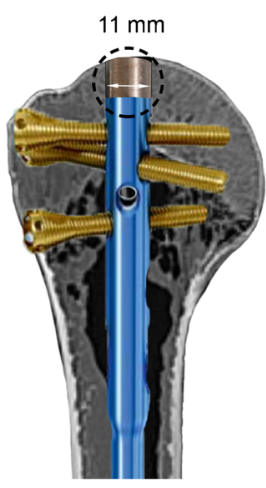

B2 
to the cylindrical axis of the mold in order to apply varus dislocating force (Fig. 3) [8,9]. Prior to potting the humeral head, two screws were inserted through the head, oriented medially to the nail to ensure rigid fixation in the PMMA.

\section{Biomechanical testing}

The humeri were biomechanically evaluated using a dynamic tensile testing machine (ElectroPuls E10000; Instron Systems, Norwood, MA, USA). Before the testing of each humerus, a cylindrical metal socket with a spherical cavity for load application (via a ball attached to the testing machine actuator) was fixed within the medial side of the proximal potting (Fig. 3). Each humerus was secured within a custom fixture at the distal potting, while ensuring the proximal potting on the humeral head was level in both anterior/posterior and medial/lateral directions. In $20^{\circ}$ of abduction, the humerus was preloaded to $15 \mathrm{~N}$, which was the minimum load for subsequent cyclic loading. Using a caliper, the gap of the osteotomy was measured at the most medial location. The specimen was then cyclically loaded between 15 and $50 \mathrm{~N}$ at a frequency of $0.25 \mathrm{~Hz}$ with the maximum load increasing stepwise by $50 \mathrm{~N}$ every 100 cycles. After completion of each load step, the osteotomy gap was re-measured. Endpoint of testing, due to failure of the fixation, was defined as an osteotomy gap closure measurement greater than or equal to $5 \mathrm{~mm}$ relative to the initial gap measurement, which appeared to be equivalent to a permanent varus displacement in preliminary experiments. Implant cut out as well as any other fracture

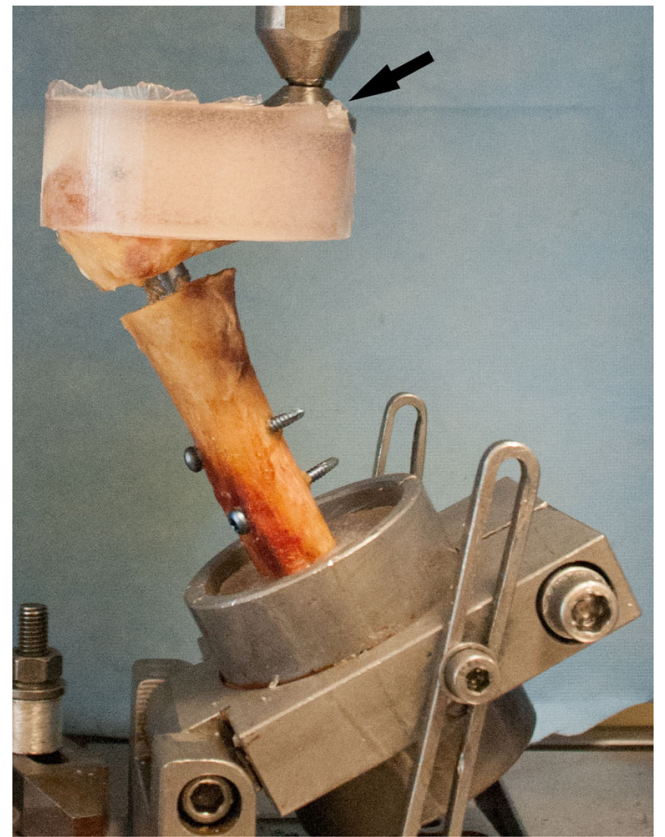

Fig. 3 Test setup: anterior view of a right humerus positioned in the testing jig, oriented in $20^{\circ}$ of abduction. A compressive two-point bending force was applied on a socket (black arrow) to apply varus dislocating force occurrence were also defined as fixation failure. Failure load was defined as the last completely sustained load magnitude during the stepwise increasing cyclic loading without failure. Following testing, all specimens were carefully freed of all potting material and examined.

\section{Statistical analysis}

To address the primary hypothesis, failure load was compared between two fixation methods of each test group separately. To reflect the fact that a failure event was defined based on measurements taken after each load step with 100 cycles, the overall load data were analyzed as an ordinal variable. Thus, medians and ranges were reported, and the Wilcoxon signedrank test was conducted. An a priori sample size calculation indicated that ten specimens per group would be sufficient to detect an effect size of $d=1$ for matched-pairs testing with $80 \%$ power. The statistical software package R ( R Development Core Team, Vienna, Austria) was used for all data analysis and statistical significance was declared for $p<0.05$ [10].

\section{Results}

In 17 of the 38 specimens, a fracture of the greater tuberosity occurred (Fig. 4a); 19 specimens failed by means of osteotomy gap closure of $>5 \mathrm{~mm}$ (Fig. $4 \mathrm{~b}$ ). Locking screw cut-through and failure of the fixture at $450 \mathrm{~N}$ were each seen in one specimen. There was no significant difference in mode of failure pattern between the different configurations of endcaps overall or in groups A or B, separately. There were also no significant differences in failure load between the specimens that failed by gap closure versus those that failed by greater tuberosity fracture overall or in groups A or B, separately.

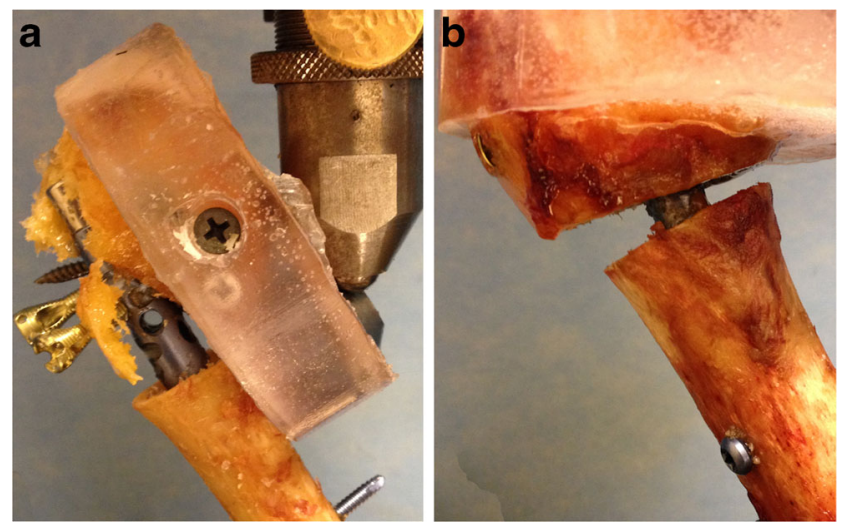

Fig. 4 Representative failure pattern of surgically prepared and tested humeri. a Anterior view on a right humerus: fracture of the greater tuberosity. b Anterior view on a left humerus: closure of the medial osteotomy gap 
In the no-PAP group $\mathrm{A}$, the specimens with a common size endcap subgroup A1 (median, $450 \mathrm{~N}$ [range, 200-1.000]) exhibited significantly higher loads to failure than the no endcap subgroup A2 (median, $325 \mathrm{~N}$ [range, 100-500]; $p=0.009$ ). In the custom-PAP group $\mathrm{B}$, maximal loading between the specimens with common sized endcap subgroup B1 (median, $450 \mathrm{~N}$ [range, 200-600]) and custom endcap subgroup B2 (median, $400 \mathrm{~N}$ [range, 200-650]; $p=0.240$ ) were not found to be statistically significant (Fig. 5). Overall, specimens were found to be osteoporotic (mean BMD, $70 \mathrm{mg}$ / $\mathrm{cm}^{3}$ [range 52-88]). Within our sample, there was no significant correlation between failure load and BMD $(r h o=0.406$, $p=0.085$ ).

\section{Discussion}

The most important finding of this study was that the "proximal anchoring point" provided significantly increased stability in straight humeral nail constructs as compared to the same setup without this additional anchoring point.

Secondary varus displacement has been described as a typical failure pattern in proximal humeral fracture treatment $[2$, 11]. The most common fracture mechanism is falling on the outstretched arm; this compressive load may severely damage the medial calcar and, hence, the medial bony mechanical support $[12,13]$. This is of particular relevance in osteoporotic bone. The anatomic reduction of the medial calcar has been shown to play a key role in this context, as it provides important support against varus displacement $[13,14]$.

Many different approaches have been proposed to overcome this severe complication. In an effort to strengthen plating constructs, screw cement augmentation [15], bony autograft or allograft augmentation [16], and additional calcar screws or blades [17] have shown promising biomechanical and clinical results. However, each of these augmentation methods bears the increased risk of infection or impending difficulties in the potentiality of surgical revision.

Theoretically, SAN seems to be an eligible treatment option in those kinds of fractures at risk for secondary varus displacement. However, there are only a few biomechanical studies available that evaluated this intramedullary implant. In 2012, Rothstock et al. [18] presented increased stability and decreased varus displacement using a supplementary "screwin-screw" configuration and advocated an additional ascending calcar screw for SAN. Contrarily, Katthagen et al. [19] showed that an additional unlocked calcar screw did not increase stability in a two-part fracture model fixed using a SAN construct.

In a clinical study, straight designed antegrade humeral nails have demonstrated superior clinical outcomes, compared to curvilinearly designed nails, with a minimum 1-year postoperative Constant Score of 83.3 versus 72.7. Complications related to rotator cuff disease were found to be significantly lower after straight nailing (34.6\% versus $73 \%$ ) [4]. Furthermore, the study demonstrated a significantly lower reoperation rate in the straight nail group (11.5\% versus $42 \%)$ [4]. In another clinical study, Hessmann et al. [20] found a mean Constant Score of 66.1 points after a mean follow-up time of six months in 17 patients with a mean age of 67.2 years. All fractures were healed, and intra-articular screw penetration and loss of reduction were each observed once.

In our study, $50 \%$ of all the specimens failed by closure of the medial gap, while $44.7 \%$ failed by fracture of the greater tuberosity. Failure of the potting and screw cut-through were each seen once. Theoretically, constructs with the PAP are more likely to fail by a fracture of the greater tuberosity due to the direct lateral force towards the cortical/subchondral humeral head. In contrast, constructs without the PAP do not directly compress the cortical/subchondral humeral head and are, therefore, expected to fail by migration of the nail inside the fatty osteoporotic cancellous substance of the humeral head to the lateral side, resulting in varus dislocation (closure
Fig. 5 Boxplots for failure load (N) for each group and endcap type. Dots represent individual data points and are jittered horizontally for clarity. *Significant difference between groups with (subgroup A1) and without (subgroup A2) the "proximal anchoring point" $(p=0.009)$
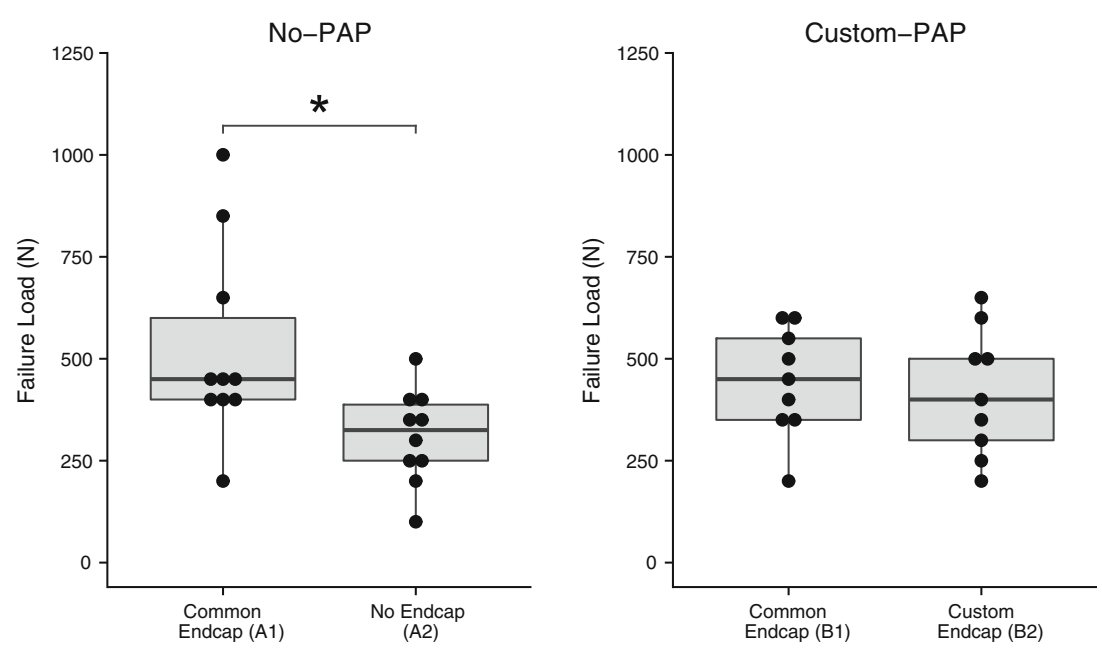
of the medial gap). However, we did not find any significant differences in failure mode patterns between the different construct configurations, nor in failure load between the specimens that failed by gap closure versus those that failed by greater tuberosity fracture overall, or in groups A or B, separately.

Accounting for up to $22 \%$, screw cutout is known to be another common complication following surgical treatment of proximal humeral fractures [21]. In our study, we did observe one screw cut-through, but no screw cutout, backing out, or any other obvious signs of implant failure. The nail/screw construct appeared to be a stable and reliable implant in this biomechanical setting.

This study has inherent limitations. This was a time-zero in vitro biomechanical model, which does not reflect the biological healing that occurs in vivo. Due to the increasing incidence of unstable proximal humeral fractures in elderly patients [22], osteoporotic humeri were used for this study. Therefore, comparable supportive characteristics of the PAP in younger humeri with potential higher BMD and stronger subchondral bone substance can only be assumed. Furthermore, 9.5-mm-sized nails were utilized in all specimens for this study. In our daily clinical practice, smaller-sized straight nails with a diameter of $8 \mathrm{~mm}$ are also frequently used. However, regardless of the nail's diameter, the manufacturer does only provide one size of endcaps $(9.5 \mathrm{~mm})$ - most likely leading to comparable biomechanical results on the PAP for smaller-sized nail diameters. As in most biomechanical investigations, simplified loading conditions were used to simulate repetitive daily activity with the arm slightly abducted. Complex loading conditions and forces generated by the various muscle and soft tissue attachments in vivo could not be taken into account in this study. Future biomechanical and clinical studies should investigate the possible benefits of the SAN compared with conventional lateral locking plate fixation for unstable proximal humeral fractures.

The PAP provided significantly increased stability in SAN constructs compared to the same setup without this additional proximal anchoring point. Varus-displacing forces to the humeral head were superiorly reduced in this setting. This study provides biomechanical evidence for the rationale of the PAP. Straight antegrade humeral nailing may be beneficial for patients undergoing surgical treatment for unstable proximal humeral fractures to decrease secondary varus displacement and thus potentially reduce revision rates.

\footnotetext{
Acknowledgements Open access funding provided by University of Innsbruck and Medical University of Innsbruck. The authors acknowledge o. Univ.-Prof. Dr. Michael Blauth for his valuable assistance with the planning of the study design. Dr. Martin Jaeger for his cooperation regarding the European Forearm Phantom (CT-based bone mineral density measurements). Barry Eckhaus and Angelica Wedell for their assistance with the photos, and Clemens Unterwurzacher for his assistance with the drawings presented in the manuscript.
}

\section{Compliance with ethical standards}

Conflict of interest and funding information This research was supported by the Steadman Philippon Research Institute. The Institute receives research support from the following entities: Smith \& Nephew Endoscopy, Inc.; Arthrex, Inc.; Siemens Medical Solutions USA, Inc.; Ossur Americas, Inc.; Opedix, Inc. This work was directly supported by outside funding (DePuy Synthes [West Chester, PA, USA] provided monetary support as well as unrestricted in-kind donations of the surgical tools utilized in this study. Grant agreement no. 13515). Peter J. Millett has received from Arthrex something of value (exceeding the equivalent of US\$ 500) not related to this manuscript or research. He is a consultant and receives payments from Arthrex and has stock options in GameReady and Vumedi. He is also a consultant for Myos. Dr. Simon Euler and Dr. Maximilian Petri had their research positions at the Steadman Philippon Research institute funded by Arthrex. Franz S. Kralinger is a consultant and receives payments from DePuy Synthes. The other authors receive or received support from the Steadman Philippon Research Institute.

Ethical approval IRB approval was not applicable. This study was performed in accordance with the ethical standards as laid down in the 1964 Declaration of Helsinki and its later amendments or comparable ethical standards.

Open Access This article is distributed under the terms of the Creative Commons Attribution 4.0 International License (http:// creativecommons.org/licenses/by/4.0/), which permits unrestricted use, distribution, and reproduction in any medium, provided you give appropriate credit to the original author(s) and the source, provide a link to the Creative Commons license, and indicate if changes were made.

\section{References}

1. Jost B, Spross C, Grehn H, Gerber C (2013) Locking plate fixation of fractures of the proximal humerus: analysis of complications, revision strategies and outcome. J Shoulder Elb Surg 22(4):542549. doi:10.1016/j.jse.2012.06.008

2. Owsley KC, Gorczyca JT (2008) Fracture displacement and screw cutout after open reduction and locked plate fixation of proximal humeral fractures [corrected]. J Bone Joint Surg Am 90-A:233240. doi:10.2106/jbjs.f.01351

3. Sproul RC, Iyengar JJ, Devcic Z, Feeley BT (2011) A systematic review of locking plate fixation of proximal humerus fractures. Injury 42(4):408-413. doi:10.1016/j.injury.2010.11.058

4. Lopiz Y, Garcia-Coiradas J, Garcia-Fernandez C, Marco F (2014) Proximal humerus nailing: a randomized clinical trial between curvilinear and straight nails. J Shoulder Elb Surg 23:369-376. doi:10. 1016/j.jse.2013.08.023

5. Euler SA, Hengg C, Kolp D, Wambacher M, Kralinger F (2014) Lack of fifth anchoring point and violation of the insertion of the rotator cuff during antegrade humeral nailing: pitfalls in straight antegrade humeral nailing. Bone Joint J 96-B(2):249-253. doi:10. 1302/0301-620x.96b2.31293

6. Hepp P, Josten C (2007) Biology and biomechanics in osteosynthesis of proximal humerus fractures. Eur J Trauma Emerg Surg 33:337-344. doi:10.1007/s00068-007-7089-2

7. Krappinger D, Roth T, Gschwentner M, Suckert A, Blauth M, Hengg C, Kralinger F (2015) Preoperative assessment of the cancellous bone mineral density of the proximal humerus using CT data. Skelet Radiol 41:299-304. doi:10.1007/s00256-011-1174-7

8. Dietz SO, Hartmann F, Schwarz T, Nowak TE, Enders A, Kuhn S, Hofmann A, Rommens PM (2012) Retrograde nailing versus 
locking plate osteosynthesis of proximal humeral fractures: a biomechanical study. J Shoulder Elb Surg 21(5):618-624. doi:10. 1016/j.jse.2011.04.013

9. Gradl G, Knobe M, Stoffel M, Prescher A, Dirrichs T, Pape HC (2013) Biomechanical evaluation of locking plate fixation of proximal humeral fractures augmented with calcium phosphate cement. J Orthop Trauma 27(7):399-404. doi:10.1016/s0020-1383(12) 70040-2

10. R Core Team (2015) A language and environment for statistical computing. R Foundation for Statistical Computing, Vienna. Available at: http://www.R-project.org/

11. Brunner F, Sommer C, Bahrs C, Heuwinkel R, Hafner C, Rillmann P, Kohut G, Ekelund A, Muller M, Audigé L, Babst R (2009) Open reduction and internal fixation of proximal humerus fractures using a proximal humeral locked plate: a prospective multicenter analysis. J Orthop Trauma 23:163-172. doi:10.1097/bot.0b013e3181920e5b

12. Hertel R, Hempfing A, Stiehler M, Leunig M (2004) Predictors of humeral head ischemia after intracapsular fracture of the proximal humerus. J Shoulder Elb Surg 13(4):427-433. doi:10.1016/j.jse. 2004.01.034

13. Kralinger F, Unger S, Wambacher M, Smekal V, Schmoelz W (2009) The medial periosteal hinge, a key structure in fractures of the proximal humerus: a biomechanical cadaver study of its mechanical properties. J Bone Joint Surg Br 91:973-976. doi:10.1302/ 0301-620x.91b7.21857

14. Lin SJ, Tsai YH, Yang TY, Shen SH, Huang KC, Lee MS (2015) Medial calcar support and radiographic outcomes of plate fixation for proximal humeral fractures. Biomed Res Int 2015:170283. doi: $10.1155 / 2015 / 170283$

15. Unger S, Erhart S, Kralinger F, Blauth M, Schmoelz W (2012) The effect of in situ augmentation on implant anchorage in proximal humeral head fractures. Injury 43:1759-1763. doi:10.1016/j. injury.2012.07.003

16. Euler SA, Hengg C, Wambacher M, Spiegl UJ, Kralinger F (2015) Allogenic bone grafting for augmentation in two-part proximal humeral fracture fixation in a high-risk patient population. Arch Orthop Trauma Surg 135:79-87. doi:10.1007/s00402-014-2128-z

17. Beirer M, Crönlein M, Venjakob AJ, Saier T, Schmitt-Sody M, Huber-Wagner S, Biberthaler P, Kirchhoff C (2015) Additional calcar support using a blade device secondary varus displacement following reconstruction of the proximal humerus: a prospective study. Eur J Med Res 20:82. doi:10.1186/s40001-015-0178-5

18. Rothstock S, Plecko M, Kloub M, Schiuma D, Windolf M, Gueorguiev B (2012) Biomechanical evaluation of two intramedullary nailing techniques with different locking options in a three-part fracture proximal humerus model. Clin Biomech (Bristol, Avon) 27:686-691. doi:10.1016/j.clinbiomech.2012.03. 003

19. Katthagen JC, Schwarze M, Bauer L, Meyer-Kobbe J, Voigt C, Hurschler C, Lill H (2015) Is there any advantage in placing an additional calcar screw in locked nailing of proximal humeral fractures? Orthop Traumatol Surg Res 101:431-435. doi:10.1016/j.otsr. 2015.01.018

20. Hessmann MH, Nijs S, Mittlmeier T, Kloub M, Segers MJ, Winkelbach V, Blauth M (2012) Internal fixation of fractures of the proximal humerus with the multiloc nail. Oper Orthop Traumatol 24:418-431. doi:10.1007/s00064-011-0085-Z

21. Schliemann B, Siemoneit J, Theisen C, Kösters C, Weimann A, Raschke MJ (2012) Complex fractures of the proximal humerus in the elderly - outcome and complications after locking plate fixation. Musculoskelet Surg 96:S3-S11. doi:10.1007/s12306-0120181-8

22. Calvo E, Morcillo D, Foruria AM, Redondo-Santamaría E, OsorioPicorne F, Caeiro JR, GEIOS-SECOT Outpatient Osteoporotic Fracture Study Group (2011) Nondisplaced proximal humeral fractures: high incidence among outpatient-treated osteoporotic fractures and severe impact on upper extremity function and patient subjective health perception. J Shoulder Elb Surg 20:795-801. doi:10.1016/j.jse.2010.09.008 